\title{
An Application of Slow Feature Analysis to the Genetic Sequences of Coronaviruses and Influenza viruses
}

\section{Anastasios Tsonis ( $\square$ aatsonis@uwm.edu )}

University of Wisconsin-Milwaukee https://orcid.org/0000-0003-4474-3423

\section{Geli Wang}

Chinese Academy of Sciences

\section{Lvyi Zhang}

Chinese Academy of Sciences

\section{Wenxu Lu}

Chinese Academy of Sciences

\section{Aristotle Kayafas}

Miami University

\section{Katia Del Rio-Tsonis}

Miami University

Primary research

Keywords: DNA complexity, Slow Feature Analysis, Coronaviruses, Influenza viruses

Posted Date: March 15th, 2021

DOI: https://doi.org/10.21203/rs.3.rs-294807/v1

License: (c) (1) This work is licensed under a Creative Commons Attribution 4.0 International License. 
1

$18 *$ Corresponding authors

Anastasios A. Tsonis (aatsonis@uwm.edu) and Katia Del Rio-Tsonis (delriok@miamioh.edu)

\section{An Application of Slow Feature Analysis to the Genetic Sequences of Coronaviruses and Influenza viruses}

Anastasios A. Tsonis ${ }^{1,2, *}$ Geli Wang ${ }^{3}$, Lvyi Zhang ${ }^{3}$, Wenxu Lu ${ }^{3}$, Aristotle Kayafas ${ }^{4}$, and Katia Del Rio-Tsonis ${ }^{4 *}$

1 Department of Mathematical Sciences, Atmospheric Sciences Group, University of WisconsinMilwaukee, Milwaukee, WI 53201, USA

${ }^{2}$ Hydrologic Research Center, San Diego, CA 92127, USA

${ }^{3}$ Key Laboratory of Middle Atmosphere and Global Environment Observation (LAGEO), Institute of Atmospheric Physics, Chinese Academy of Sciences, Beijing 100029, China

${ }^{4}$ Department of Biology and Center for Visual Sciences at Miami University, Oxford, OH 45056, USA

\section{Abstract}

\section{Background}

24 Mathematical approaches have for decades used to probe the structure of DNA sequences. This

25 has led to the development of Bioinformatics. In this exploratory work, a novel mathematical

26 method is applied to probe the DNA structure of two related viral families; those of

27 coronaviruses and those of influenza viruses. The coronaviruses are SARS-CoV-2, SARS-CoV-

28 1, and MERS. The influenza viruses include H1N1-1918, H1N1-2009, H2N2-1957, and H3N2-

291968. 
31 The mathematical method used is the Slow Feature Analysis (SFA), a rather new but promising

32 method to delineate complex structure in DNA sequences.

\section{Results}

34 The analysis indicates that the DNA sequences exhibit an elaborate and convoluted structure

35 akin to complex networks. We define a measure of complexity and show that each DNA

36 sequence exhibits a certain degree of complexity within itself, while at the same time there exists

37 complex inter-relationships between the sequences within a family and between the two families.

38 From these relationships, we find evidence, especially for the coronavirus family, that increasing

39 complexity in a sequence is associated with higher transmission rate but with lower mortality.

\section{Conclusions}

41 The complexity measure defined here may hold a promise and could become a useful tool in the

42 prediction of transmission and mortality rates in future new viral strains.

44 Key words: DNA complexity, Slow Feature Analysis, Coronaviruses, Influenza viruses

\section{Background}

47 Since the early 1970s, scientists have attempted to discover some kind of order or hidden

48 structures in DNA sequences. With the advent of sequencing techniques in the late 1970s,

49 scientists had the opportunity to probe nucleic acid sequences for such order $[1,2,3]$. Soon,

50 mathematical approaches were employed to shed light in this endeavor, leading to the full-blown

51 field of Bioinformatics [4, 5, 6, 7]. We report, for the first time, the application of Slow Feature

52 Analysis (SFA) to genetic sequences. SFA is a procedure for extracting slowly varying, driving

53 signals from a given nonstationary time series, and is used here to delineate signals or structure

54 in DNA sequences, which would not otherwise be detected. Descriptions of this procedure, 
55 which has been successfully applied in many scientific areas have been reported previously in 56 detail $[8,9,10]$.

\section{Methods}

60 SFA is an approach that is designed to optimally identify low-frequency behavior in a time

61 series, thereby delineating its complex structure more effectively. This analysis is rooted,

62 theoretically, in the time-embedding theorems. In this method, a one-dimensional time series is

63 embedded in a multi-dimensional space consisting of the original time series and lagged copies

64 thereof. SFA further uses a nonlinear expansion to map this multi-dimensional input signal onto

65 an even larger feature space, and then solves a linear problem to find a linear combination of

66 feature-space variables that minimizes their time derivative (rate of change) [11]. The objective

67 of SFA is to find the optimally filtered signals that vary as slowly as possible, but still carry

68 significant information. To ensure this, the output signals need to be uncorrelated and have unit

69 variance [12]. This approach has been successfully applied in many areas, including climate

70 science $[13,14]$.

71

72 In mathematical terms [8] the goal of SFA is, given an $n$-dimensional input signal $\mathbf{x}(t)$, to find a

73 set of real-valued input-output functions $g_{\mathrm{j}}(\mathbf{x})$ such that the output signals

74

75

76

77

78

79 under the constraints

80

81

82

$$
y_{\mathrm{j}}(\mathrm{t}):=g_{\mathrm{j}}(\mathbf{x}(\mathrm{t}))
$$

$$
\text { minimize } \Delta\left(y_{i}\right):=<\dot{y}_{j}^{2}>_{t}
$$

$$
\left\langle y_{\mathrm{j}}\right\rangle_{\mathrm{t}}=0
$$

(zero mean),

$$
\left\langle y_{\mathrm{j}}^{2}\right\rangle_{\mathrm{t}}=1
$$

(unit variance),

$$
\forall \mathrm{i}\left\langle\mathrm{j}:\left\langle y_{\mathrm{i}} y_{\mathrm{j}}\right\rangle_{\mathrm{t}}=0\right. \text { (decorrelation and order) }
$$

83 with $\langle\cdot\rangle_{\mathrm{t}}$ and $\dot{y}$ indicating temporal averaging and the derivative of $y$, respectively. 
85 The $\Delta$-value is a measure of the temporal slowness of the signal $y(t)$. It is given by the mean

86 square of the signal's time derivative. Small $\Delta$-values correspond to slowly varying signals. The

87 first two constraints avoid the trivial constant solution, while the last constraint guarantees that

88 the output functions $g_{\mathrm{j}}$ are distinct and hence extract different information from the input signal.

89 For a tutorial on this method the reader could consult references [8] or a more recent presentation

90 in [15]. In that tutorial, a simple example of a two-dimensional input signal $x_{1}(t)=\sin (t)+\cos (11 t)^{2}$

91 and $x_{2}(t)=\cos (11 t)$ is considered. Both components are quickly varying, but hidden in the signal

92 is the slowly varying 'feature' $y(t)=x_{1}(t)-x_{2}(t)^{2}=\sin (t)$, which can be extracted with a polynomial

93 of degree two, namely $h(\mathbf{x})=x_{1}-x_{2}{ }^{2}$.

95 In the situation with one observable (time series of some variable) from an unknown system

96 where the actual state space is not known (as is the case here), embedding is necessary (and

97 essential) to delineate the underlying dynamics much like in attractor reconstructions. The SFA

98 algorithm can be summarized as follows. Consider a time series $\{x(t)\}_{t=t_{1}, \ldots, t_{n}}$, where $t$ denotes

99 time and $n$ indicates the length of the time series. First, we embed $\{x(t)\}$ into an $m$-dimensional

100 state space using time delayed copies of $x(t)$ :

101

102

$$
\mathbf{X}(t)=\left\{x_{1}(t), x_{2}(t), \ldots, x_{m}(t)\right\}_{t=t_{1}, \ldots, t_{N}}
$$

103

where $x_{1}(t)=x(t) ; x_{2}(t)=x_{1}(t-\tau) ; x_{3}(t)=x_{1}(t-2 \tau)$, and so on, $\tau$ is the delay, and $N=$ $105 n-m+1$. Then, nonlinear expansions (usually second-order polynomials) are used to generate a $106 k$-dimensional function state space:

$$
\mathbf{H}(t)=\left\{x_{1}(t), \ldots, x_{m}(t), x_{1}^{2}(t), \ldots, x_{1}(t) x_{m}(t), \ldots, x_{m-1}^{2}(t), \ldots, x_{m}^{2}(t)\right\}_{t=t_{1}, \ldots, t_{N}},
$$

$$
k=m+m(m+1) / 2 .
$$


114 The expanded signal $\mathbf{H}(t)$ is then centered and normalized to zero mean and unit variance. This 115 process is referred to as whitening or sphering. Thus, we have

116

117

118

119

120

121

122

123

124

125

126

127

128

129

130

131

132

133

134

135

136

137

138

139

140

141

142 corresponding to the smallest eigenvalue $\lambda_{1}$. Here

Using Schmidt algorithm, $\mathbf{H}^{\prime}(t)$ is orthogonized into:

where the transformed signal matrix $\mathbf{Z}$ is column orthogonal:

$$
\overline{h_{\jmath}^{\prime}}=0 \text { (zero mean), }
$$

$$
h_{j}^{\prime} h_{j}^{\prime T}=1 \text { (unit variance), }
$$

$$
h_{j}^{\prime}(t)=\left[h_{j}(t)-\bar{h}_{j}\right] / S, \text { and } S=\frac{1}{k} \sqrt{\sum_{j=1}^{k}\left(h_{j}(t)-\bar{h}\right)^{2}} .
$$

$$
\bar{z}_{l}(t)=\bar{z}_{J}(t)=0, z_{i}^{T}(t) \cdot z_{j}(t)=0, z_{j}^{T}(t) \cdot z_{j}(t)=1
$$

The final step of SFA is to find the set of coefficients $\left(a_{1}, a_{2}, \ldots, a_{k}\right)$ such that the time series

$$
y(t)=a_{1} z_{1}(t)+a_{2} z_{2}(t)+\ldots+a_{k} z_{k}(t)
$$




$$
\dot{\mathbf{Z}}(t)=\left\{\dot{z_{1}}(t), \dot{z_{2}}(t), \ldots, \dot{z_{k}}(t)\right\}_{t=t_{1}, \ldots, t_{N}}
$$

145 and

$$
\dot{z}_{J}\left(t_{i}\right)=z_{j}\left(t_{i+1}\right)-z_{j}\left(t_{i}\right)
$$

148 Using $\mathbf{W}_{1}$, the optimally filtered slow-feature signal (also known as a driving force factor) can be 149 written as:

$$
y(t)=r \mathbf{W}_{1} \cdot \mathbf{Z}(\mathrm{t})+\mathrm{c},
$$

where $r$ and $c$ are constants derived to best match $y(t)$ and the original time series $x(t)$.

155 Once the optimally filtered (low-frequency) SFA signal has been identified, its significant periodicities can be found from the time-averaged wavelet power spectrum. Wavelet analysis has

157 been widely used to analyze localized structures and spectral properties of time series. For 158 example, [16] provides a detailed description of the wavelet analysis, along with a very useful toolkit to conduct step-by-step wavelet analysis, including a statistical significance test based on the red-noise surrogate data (see http://paos.colorado.edu/research/wavelets/). We here used the

161 Morlet wavelet with the wavenumber set to 4 to match the smoothness of the SFA-derived slow162 feature signal, focusing, once again, on the spectral peaks statistically significant at the 5\% level. 163 Note also that SFA is applicable to non-stationary data, so no data pre-processing is required.

165 The combination of the SFA and wavelet analyses we use in the present study has been shown to 166 be more effective in diagnosing low-frequency periodicities in data sets of a limited length than 167 direct spectral analysis methods. Note that the driving force may not necessarily consist of just 168 one component, but several components, which, as we will see below, correspond to forcings or 169 signals at certain time scales. The success of SFA in delineating these slow signals lies in the fact 170 that embedding the time series in high enough dimensions and the subsequent dynamical 171 procedure, removes the noise and small-scale features that may obscure or suppress those slow 172 signals, thereby delineating more accurately the complex structure of a sequence. 


\section{Analysis and results}

175 We first analyzed the DNA sequences from three viruses from the same family: SARS-CoV-2,

176 SARS-CoV-1, and MERS. Those sequences are approximately 30,450 bases long, and part of the

177 now world-infamous coronavirus family. Since a nucleotide sequence is a string of the bases A,

$178 \mathrm{~T}$ (U in RNA), C, and G, we first transformed it to a time series of integers in the interval [1-4]

179 (i.e. $A \rightarrow 1, T / U \rightarrow 2, C \rightarrow 3, G \rightarrow 4$ ). Then, we applied SFA, and once we had the SFA signal

180 (which as we mentioned above may be comprised of several components), we extracted the SFA

181 components by wavelet analysis ${ }^{1}$. Figure 1 shows the SFA signal for SARS-CoV-2 virus for

$182 \mathrm{~m}=15$ and $\tau=1$. As explained above, this signal is normalized to zero mean and unit variance.

183 Figure 2 shows the wavelet of the time series in Figure 1. In order to extract the peak "periods"

184 of the driving force signal, we used the Morlet wavelet to compute the time-averaged power

185 spectrum of the wavelet transform [16]. The black solid line in Figure 3 is the time-averaged

186 power spectrum of the wavelet transform of the driving force, and the dashed line represents the

187 95\% confidence level, estimated using AR-1 surrogate data [16]. The dots show the periods of

188 the oscillatory components of the driving force that are significant above the $95 \%$ level.

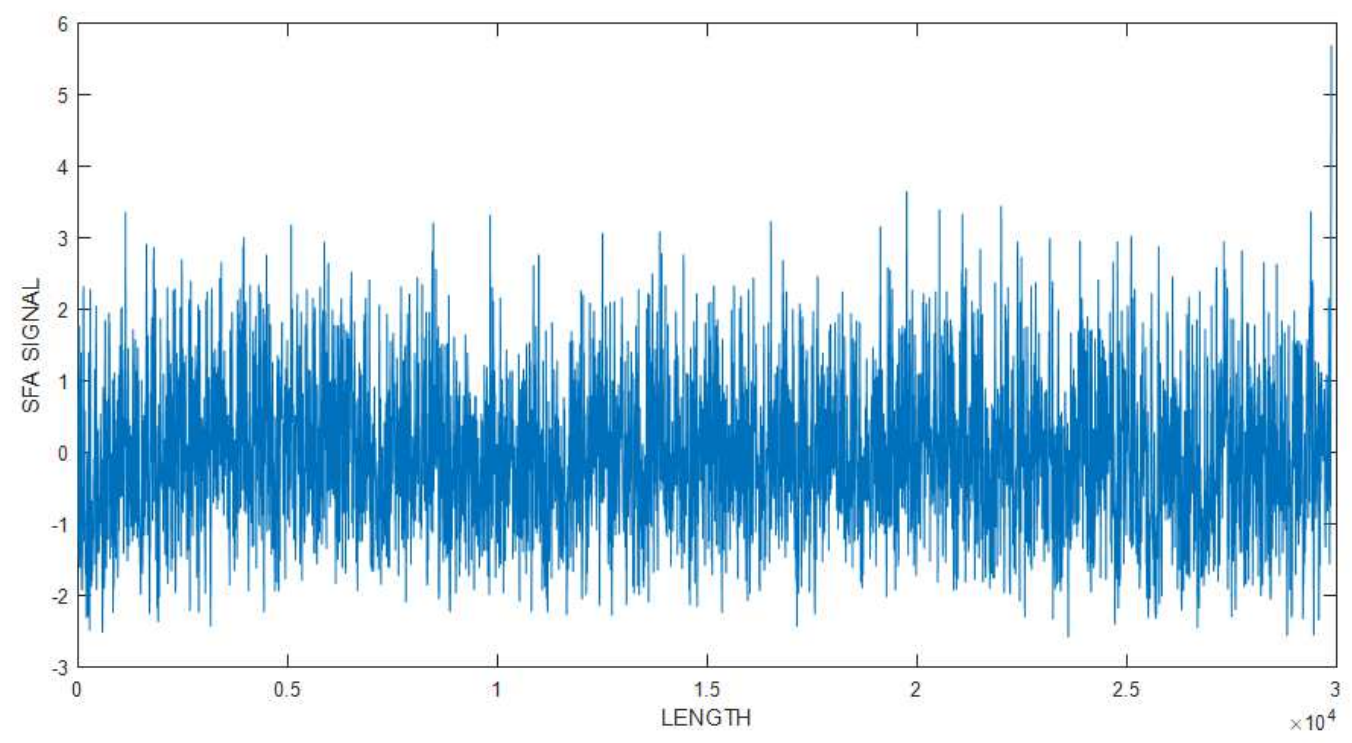

\footnotetext{
${ }^{1}$ Note that the above transformation of $\mathrm{A} \rightarrow 1, \mathrm{~T} / \mathrm{U} \rightarrow 2, \mathrm{C} \rightarrow 3, \mathrm{G} \rightarrow 4$ may, depending on frequency distribution of $\mathrm{A}, \mathrm{T} / \mathrm{U}, \mathrm{C}, \mathrm{G}$ in the sequence, result in a nonstationary time series. However, unlike other spectral methods, SFA is not affected by nonstationarity in the data.
} 
190 Figure 1: The SFA signal of the DNA sequence of SARS-CoV-2. Note the oscillatory components 191 at many scales.

192

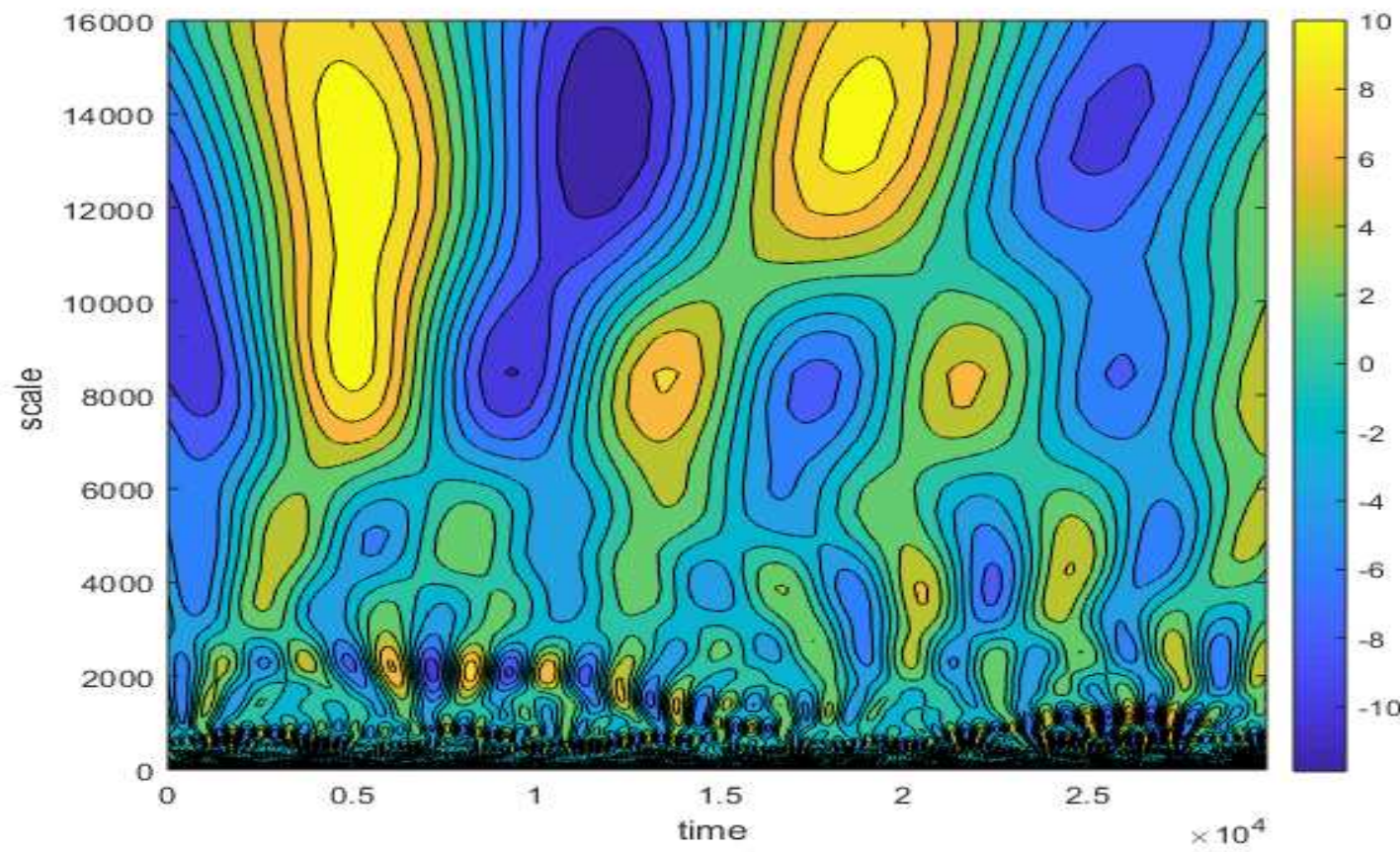

193

194 Figure 2: The wavelet of the signal extracted from Figure 1.

195

196

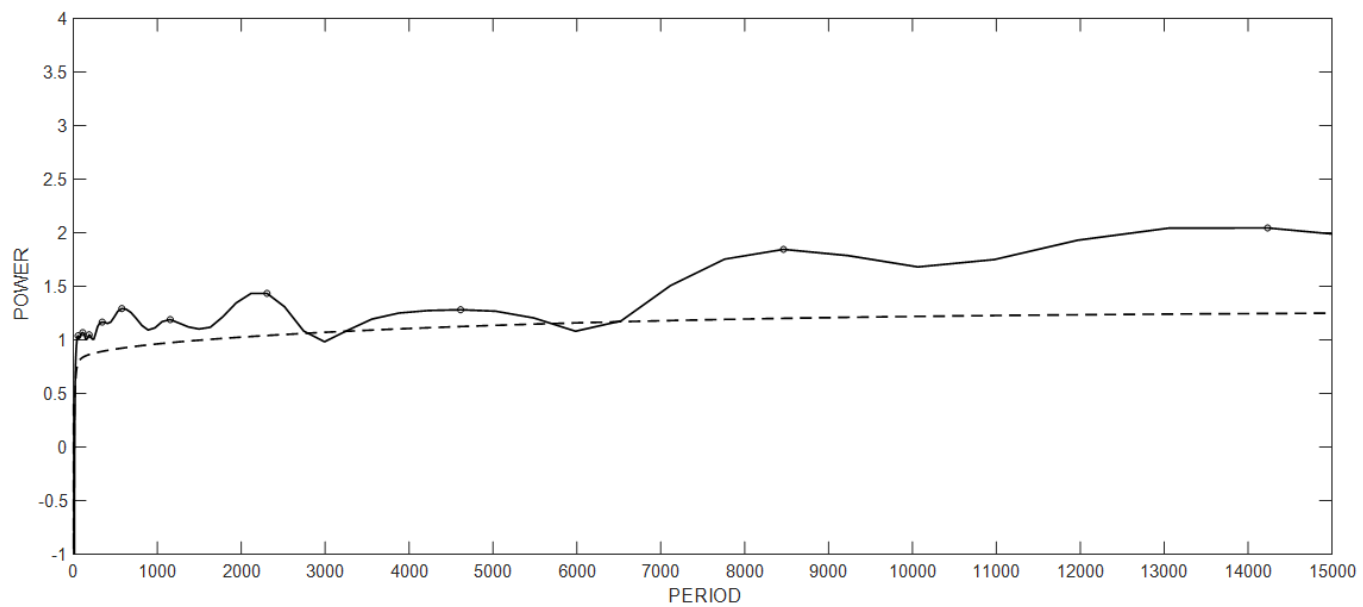


198 Figure 3: The time-averaged power spectrum of the wavelet transform extracted from Figure 2.

199 The dashed line represents the 95\% confidence level. The dots show the periods of the oscillatory

200 components of the driving force that are significant above the 95\% level.

201

202 The significant peak periodicities for SARS-CoV-2 are $^{2}$ :

203

$204 \mathrm{P}_{1}=55.5956928123500$

$205 \mathrm{P}_{2}=111.191385624700$

$206 \quad \mathrm{P}_{3}=187.000875157807$

$207 \mathrm{P}_{4}=342.961117205042$

$208 \quad \mathrm{P}_{5}=576.789548058258$

$209 \mathrm{P}_{6}=1153.57909611652$

$210 \quad \mathrm{P}_{7}=2307.15819223303$

$211 \quad \mathrm{P}_{8}=4614.31638446607$

$212 \quad \mathrm{P}_{9}=8462.69356236189$

$213 \mathrm{P}_{10}=14232.4973599616$

214

215

216 Given the above periodicities recovered from SARS-CoV-2, we next construct Table 1, which

217 shows the ratios between these peaks. We observe the following EXACT relations between peak 218 periods:

219

$220 \mathrm{P}_{2}=2 \mathrm{P}_{1}$

$221 \quad \mathrm{P}_{10}=128 \mathrm{P}_{2}$

$222 \mathrm{P}_{10}=256 \mathrm{P}_{1}$

$223 \quad \mathrm{P}_{8}=2 \mathrm{P}_{7}$

$224 \mathrm{P}_{8}=4 \mathrm{P}_{6}$

$225 \quad \mathrm{P}_{8}=8 \mathrm{P}_{5}$

\footnotetext{
${ }^{2}$ Note here that, before we applied SFA to actual DNA sequences, and in order to test the efficiency of SFA when the time series is a string of integers, we considered artificial sequences of known periodicities. SFA was able to reproduce the known periodicities.
} 
$226 \quad \mathrm{P}_{7}=2 \mathrm{P}_{6}$

$227 \quad \mathrm{P}_{7}=4 \mathrm{P}_{5}$

$228 \quad \mathrm{P}_{6}=2 \mathrm{P}_{5}$

229

230 And the following almost exact relationships based on the criterion:

231

232

$\mid \mathrm{P}$-nearest integer $\mid /$ nearest integer $<0.25 \%$

233

234

235

\begin{tabular}{|c|c|c|c|c|c|c|c|c|c|c|}
\hline Period & P1 & P2 & P3 & P4 & P5 & P6 & P7 & P8 & $\mathrm{Pg}$ & P10 \\
\hline P1 & 1 & 2 & 3.363585661 & 6.168843302 & 10.37471644 & 20.74943287 & 41.498866575 & 82.9977315 & 152.2185107 & 256 \\
\hline P2 & & 1 & 1.681792831 & 3.084421651 & 5.187358219 & 10.37471644 & 20.74943287 & 41.49886575 & 76.10925536 & 128 \\
\hline P3 & & & 1 & 1.834008086 & 3.084421651 & 6.168843302 & 12.3376866 & 24.67537321 & 45.254834 & 76.10925536 \\
\hline P4 & & & & 1 & 1.681792831 & 3.363585661 & 6.727171322 & 13.45434264 & 24.67537321 & 41.49886575 \\
\hline P5 & & & & & 1 & 2 & 4 & 8 & 14.67206469 & 24.67537321 \\
\hline P6 & & & & & & 1 & 2 & 4 & 7.336032346 & 12.3376866 \\
\hline P7 & & & & & & & 1 & 2 & 3.668016173 & 6.168843302 \\
\hline P8 & & & & & & & & 1 & 1.834008086 & 3.084421651 \\
\hline P9 & & & & & & & & & & 1.681792831 \\
\hline P10 & & & & & & & & & & \\
\hline
\end{tabular}

236

237 Table 1: Ratios between the peaks in (1)

238

239

$240 \quad \mathrm{P}_{9}=152 \mathrm{P}_{1}$

$241 \quad \mathrm{P}_{9}=76 \mathrm{P}_{2}$

$242 \mathrm{P}_{10}=76 \mathrm{P}_{3}$

$243 \quad \mathrm{P}_{8}=83 \mathrm{P}_{1}$

244

245

246 Keeping only those relationships, we remain with Table 2, which could be thought as portraying

247 the degree of structure or complexity in the SARS-CoV-2 sequence. We observe in the exact

248 relationships, multiples of a power of 2, and in the almost exact relationships multiples of 19

$249(152=2 \times 76=8 \times 19)$ and 83. Clearly, a sophisticated, and rather convoluted structure, with 
250 numerous processes embedded in the sequence, is present. Keep in mind that the factors 19 and 25183 (odd numbers) will appear in the rest of the sequences studied here. We define the number of 252 entries above the diagonal in Table 2 as the degree complexity, C. In this case $\mathrm{C}=13$.

253

254

\begin{tabular}{|r|r|r|r|r|r|r|r|r|r|r|}
\hline Period & P1 & P2 & P3 & P4 & P5 & P6 & P7 & P8 & P9 & P10 \\
\hline P1 & 1 & 2 & & & & & & 83 & 152 & 256 \\
\hline P3 & & 1 & & & & & & & 76 & 128 \\
\hline P4 & & 1 & & & & & & & 76 \\
\hline P5 & & & 1 & & & & & & \\
\hline P6 & & & & 1 & 2 & 4 & 8 & \\
\hline P8 & & & & & 1 & 2 & 4 & \\
\hline P9 & & & & & & 1 & 2 & \\
\hline P10 & & & & & & 1 & \\
\hline
\end{tabular}

Table 2: Same as Table 1 but keeping only the exact and almost exact relationships see

258 relationships (2) and (3).

261 In the Supplementary material, Figures S1, S2, S3 are similar to Figures 1, 2, 3, and Tables ST1, 262 ST2 are similar to Tables 1, 2 but for SARS-CoV-1. Figures S4, S5, S6 are similar to Figures 1, 263 2, 3, and Tables ST3, ST4 are similar to Tables 1, 2 but for MERS.

266 According to Figures S3 the peak periods for SARS-CoV-1 are,

$268 \quad \mathrm{P}_{1}=50.9814750936898$

$269 \quad \mathrm{P}_{2}=111.191385624700$

$270 \quad \mathrm{P}_{3}=528.918347647618$

$271 \quad \mathrm{P}_{4}=748.003500631229$

$272 \quad \mathrm{P}_{5}=2115.67339059047$

$273 \quad \mathrm{P}_{6}=3558.12433999040$ 
$274 \quad \mathrm{P}_{7}=8462.69356236189$

$275 \quad \mathrm{P}_{8}=13051.2576239846$

276

277 and according to Tables ST1 and ST2 we now have five exact periodicities

278

$279 \quad \mathrm{P}_{5}=4 \mathrm{P}_{3}$

$280 \mathrm{P}_{6}=32 \mathrm{P}_{2}$

$281 \quad \mathrm{P}_{7}=4 \mathrm{P}_{5}$

$282 \quad \mathrm{P}_{7}=16 \mathrm{P}_{3}$

$283 \mathrm{P}_{8}=256 \mathrm{P}_{1}$

284

285 And three almost exact

286

$287 \quad \mathrm{P}_{5}=19 \mathrm{P}_{2}$

$288 \mathrm{P}_{7}=166 \mathrm{P}_{1}$

$289 \quad \mathrm{P}_{7}=76 \mathrm{P}_{2}$

290

291 Again here, we observe in the exact relationships, multiples of a power of 2, and in the almost

292 exact between $P_{7}$ and $P_{1}, P_{7}$ and $P_{2}$ and between $P_{5}$ and $P_{2}$. Note again the multiples of 19 and 83 .

293 Note also that from the almost exact relationships it follows that $\mathrm{P}_{7} / \mathrm{P}_{5}=4$, which is one of the

294 exact relationships. Here the degree of complexity is $\mathrm{C}=8$.

295

296 According to Figures S6 the peak periods for MERS are

297

298

$299 \quad \mathrm{P}_{1}=101.962950187380$

$300 \quad \mathrm{P}_{2}=132.229586911904$

$301 \quad \mathrm{P}_{3}=242.510131659000$

$302 \quad \mathrm{P}_{4}=628.993462278030$

$303 \quad \mathrm{P}_{5}=1779.06216999520$

$304 \quad \mathrm{P}_{6}=2515.97384911212$ 
$306 \quad \mathrm{P}_{8}=8462.69356236189$

$307 \quad \mathrm{P}_{9}=13051.2576239846$

308

309 and according to Tables ST3 and ST4 we now have three exact periodicities

310

$311 \mathrm{P}_{9}=128 \mathrm{P}_{1}$

$312 \mathrm{P}_{8}=64 \mathrm{P}_{2}$

$313 \quad \mathrm{P}_{6}=4 \mathrm{P}_{4}$

314

315 and three almost exact relationships

316

$317 \mathrm{P}_{8}=83 \mathrm{P}_{1}$

$318 \mathrm{P}_{7}=19 \mathrm{P}_{3}$

$319 \mathrm{P}_{6}=19 \mathrm{P}_{2}$

320

$321 \mathrm{P}_{9}, \mathrm{P}_{8}, \mathrm{P}_{6}$ multiples (again in a power of 2) of $\mathrm{P}_{1}, \mathrm{P}_{2}, \mathrm{P}_{4}$ (ordered in a bottom-top "symmetric"

322 way), $\mathrm{P}_{6}, \mathrm{P}_{7}$ and $\mathrm{P}_{8}$ are multiples of $\mathrm{P}_{2}, \mathrm{P}_{3}$, and $\mathrm{P}_{1}$ but not of 2 , but again of 19 and 83 (it is

323 interesting to note that the odd multiples of 19 and 83 appear in all three sequences). Here the

324 degree of complexity is $\mathrm{C}=6$

325

326 By comparing Tables 2, ST2, and ST4 (and their associated C), one may argue that there is more 327 embedded complexity and intricate patterning in SARS-CoV-2 than SARS-CoV-1 and MERS.

\section{Other important relationships}

332 Keeping in mind that all three sequences belong to the same coronavirus family, there are

333 similarities and inter-relationships between the sequences. For example, it is easy to observe that: 334

$\mathrm{P}_{7}$ (MERS) same as $\mathrm{P}_{8}(\mathrm{SARS}-\mathrm{C} 0 \mathrm{~V}-2)$ 
$\mathrm{P}_{8}$ (MERS) same as $\mathrm{P}_{9}(\mathrm{SARS}-\mathrm{COV}-2)$

$337 \mathrm{P}_{8}(\mathrm{MERS})$ same as $\mathrm{P}_{7}$ (SARS-COV-1)

$338 \mathrm{P}_{9}(\mathrm{MERS})$ same as $\mathrm{P}_{8}(\mathrm{SARS}-\mathrm{COV}-1)$

$339 \mathrm{P}_{2}(\mathrm{SARS}-\mathrm{CoV}-2)$ same as $\mathrm{P}_{2}(\mathrm{SARS}-\mathrm{CoV}-1)$

$340 \mathrm{P}_{9}(\mathrm{SARS}-\mathrm{CoV}-2)$ same as $\mathrm{P}_{7}(\mathrm{SARS}-\mathrm{CoV}-1)$

342 In general, SFA reveals a consistent picture between these sequences with very intricate structure

343 with details at many scales, indicating a very elaborate and sophisticated embedded processes,

344 with complexity increasing from MERS to SARS-CoV-1 to SARS-CoV-2.

Extension of the analysis to the influenza viruses of H1N1-1918, H1N12009, H2N2-1957, and H3N2-1968

In an effort to provide further support for the efficiency and consistency of SFA in the analysis

of nucleotide sequences, we consider four other viral sequences from a different viral family, that

352 of the influenza viruses or the Orthomyxoviridae family [17].

354 In the Supplementary material, Figures S7, S8, and S9 and Tables ST5 and ST6 correspond to 355 H1N1-1918 and are similar to Figures 1, 2, and 3 and Tables 1 and 2. Figures S10, S11, and S12 356 and Table ST7 and ST8 correspond to H1N1-2009 and are again similar to Figures 2 and 3 and 357 Table 2. Figures S13, S14, and S15 and Tables ST9 and ST10 correspond to H2N2-1957 and are 358 similar to Figures 2 and 3 and Table 2. The same goes for Figures S16, S17, and S18 and Tables 359 ST11 and ST12, which correspond to H3N2-1968. From these Figures and Tables, it follows 360 that:

$364 \quad \mathrm{P}_{1}=60.6275329147499$

$365 \quad \mathrm{P}_{2}=157.248365569507$ 


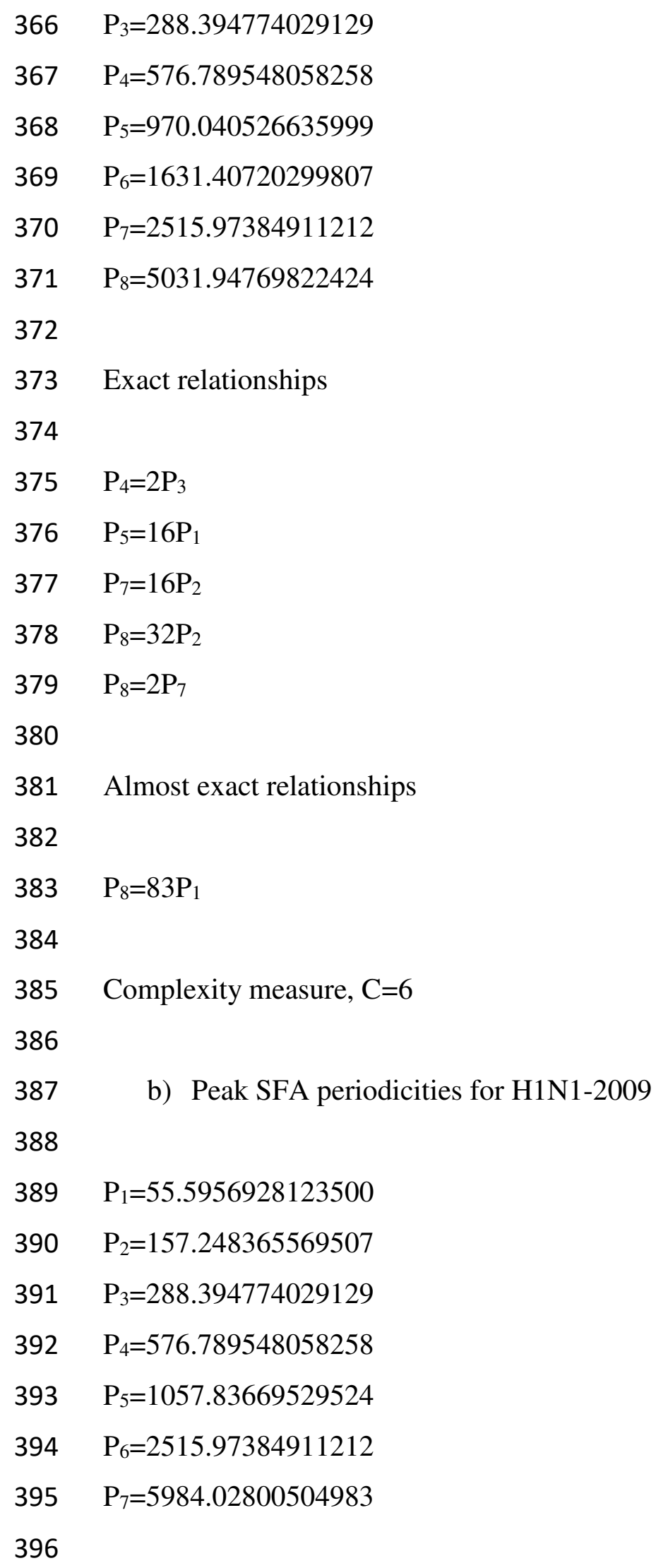

384

385 Complexity measure, $\mathrm{C}=6$

386

387

b) Peak SFA periodicities for H1N1-2009

388

$389 \quad \mathrm{P}_{1}=55.5956928123500$

$390 \quad \mathrm{P}_{2}=157.248365569507$

$391 \quad \mathrm{P}_{3}=288.394774029129$

$392 \quad \mathrm{P}_{4}=576.789548058258$

$393 \quad \mathrm{P}_{5}=1057.83669529524$

$394 \quad \mathrm{P}_{6}=2515.97384911212$

$395 \quad \mathrm{P}_{7}=5984.02800504983$

396 
397 Exact relationships

398

$399 \quad \mathrm{P}_{4}=2 \mathrm{P}_{3}$

$400 \quad \mathrm{P}_{6}=16 \mathrm{P}_{2}$

401

402 Almost exact relationships (note $38=2 \times 19$ )

403

$404 \quad \mathrm{P}_{5}=19 \mathrm{P}_{1}$

$405 \quad \mathrm{P}_{7}=38 \mathrm{P}_{2}$

406

407 Complexity measure, $\mathrm{C}=4$

408

409

c) Peak SFA periodicities for H2N2-1957

410

$411 \quad \mathrm{P}_{1}=72.0986935072823$

$412 \quad \mathrm{P}_{2}=203.925900374759$

$413 \quad \mathrm{P}_{3}=288.394774029129$

$414 \quad \mathrm{P}_{4}=576.789548058258$

$415 \quad \mathrm{P}_{5}=889.531084997600$

$416 \quad \mathrm{P}_{6}=2515.97384911212$

$417 \quad \mathrm{P}_{7}=5984.02800504983$

418

419 Exact Relationships

420

$421 \quad \mathrm{P}_{3}=4 \mathrm{P}_{1}$

$422 \quad \mathrm{P}_{4}=8 \mathrm{P}_{1}$

$423 \quad \mathrm{P}_{4}=2 \mathrm{P}_{3}$

424

425 Almost exact relationships

426

$427 \quad \mathrm{P}_{7}=83 \mathrm{P}_{1}$ 
429 Complexity measure, $\mathrm{C}=4$

430

432

$433 \quad \mathrm{P}_{1}=72.0986935072823$

$434 \quad \mathrm{P}_{2}=187.000875157807$

$435 \mathrm{P}_{3}=628.993462278030$

$436 \quad \mathrm{P}_{4}=889.531084997600$

$437 \quad \mathrm{P}_{5}=2515.97384911212$

$438 \quad \mathrm{P}_{6}=5487.37787528068$

439

440 Exact relationships

441

$442 \quad \mathrm{P}_{5}=4 \mathrm{P}_{3}$

443

444 Almost exact periodicities

445

$446 \mathrm{P}_{6}=76 \mathrm{P}_{1}$ (note $\left.76=2 \times 38=4 \mathrm{X} 19\right)$

447

448 Complexity measure, $\mathrm{C}=2$

449

450 Inter-relationships

451

452 As in the case of the coronaviruses, the influenza virus sequence analysis also revealed plenty of 453 inter-relationships as expected, since the four viruses belong to the same family.

454

$455 \quad \mathrm{P}_{2}(\mathrm{H} 1 \mathrm{~N} 1-1918)=\mathrm{P}_{2}(\mathrm{H} 1 \mathrm{~N} 1-2009)$

$456 \quad \mathrm{P}_{3}(\mathrm{H} 1 \mathrm{~N} 1-1918)=\mathrm{P}_{3}(\mathrm{H} 1 \mathrm{~N} 1-2009)=\mathrm{P}_{3}(\mathrm{H} 2 \mathrm{~N} 2-1957)$

$457 \quad \mathrm{P}_{4}(\mathrm{H} 1 \mathrm{~N} 1-1918)=\mathrm{P}_{4}(\mathrm{H} 1 \mathrm{~N} 1-2009)=\mathrm{P}_{4}(\mathrm{H} 2 \mathrm{~N} 2-1957)$

$458 \quad \mathrm{P}_{7}(\mathrm{H} 1 \mathrm{~N} 1-1918)=\mathrm{P}_{6}(\mathrm{H} 1 \mathrm{~N} 1-2009)=\mathrm{P}_{6}(\mathrm{H} 2 \mathrm{~N} 2-1957)=\mathrm{P}_{5}(\mathrm{H} 3 \mathrm{~N} 2-1968)$ 
$\mathrm{P}_{1}(\mathrm{H} 2 \mathrm{~N} 2-1957)=\mathrm{P}_{1}(\mathrm{H} 3 \mathrm{~N} 2-1968)$

$\mathrm{P}_{7}(\mathrm{H} 1 \mathrm{~N} 1-2009)=\mathrm{P}_{7}(\mathrm{H} 2 \mathrm{~N} 2-1957)$

461

462 Interestingly, we found that many relationships exist between the two viral families investigated

463 here. If we compare the results in this section to the previous section, we can infer that:

$\mathrm{P}_{1}$ (SARS-CoV-2) $=\mathrm{P}_{1}(\mathrm{H} 1 \mathrm{~N} 1-2009)$

$466 \quad \mathrm{P}_{5}(\mathrm{SARS}-\mathrm{CoV}-2)=\mathrm{P}_{4}(\mathrm{H} 1 \mathrm{~N} 1-1918)=\mathrm{P}_{4}(\mathrm{H} 1 \mathrm{~N} 1-2009)=\mathrm{P}_{4}(\mathrm{H} 2 \mathrm{~N} 2-1957)$

$467 \quad \mathrm{P}_{3}(\mathrm{SARS}-\mathrm{CoV}-2)=\mathrm{P}_{2}(\mathrm{H} 3 \mathrm{~N} 2-1968)$

$\mathrm{P}_{6}(\mathrm{MERS})=\mathrm{P}_{7}(\mathrm{H} 1 \mathrm{~N} 1-1918)=\mathrm{P}_{6}(\mathrm{H} 1 \mathrm{~N} 1-2009)=\mathrm{P}_{6}(\mathrm{H} 2 \mathrm{~N} 2-1957)=\mathrm{P}_{5}(\mathrm{H} 3 \mathrm{~N} 2-1968)$

$\mathrm{P}_{4}(\mathrm{MERS})=\mathrm{P}_{3}(\mathrm{H} 3 \mathrm{~N} 2-1968)$

471 More on this is discussed next.

\section{Discussion and Conclusions}

475

476 If we consider the peak SFA periodicities from a sequence as nodes of a community, and their

477 relationships as links between the nodes, then, a visualization of the results for the SARS-CoV-2

478 community would look like the top left panel of Figure 4. Since there are 10 peak periodicities,

479 we have 10 nodes. Then, from equations 2 and 3 we have 13 (recall that $\mathrm{C}=13$ ) links between

480 them (showing in blue). The rest of the panels correspond to the rest of the sequences in both

481 families. The red lines give the links between the communities within a family (from equation 8

482 for the coronavirus family and from equation 17 for the influenza family). The black lines are the

483 links between the two families (equation 18). This picture is a perfect example of complex

484 networks, which are often characterized by a community structure, where in each community the

485 nodes are connected in a certain way (meaning the community obeys its own dynamics), but

486 where there exist also some connections (or interactions) between the communities (see for

487 example, $[18,19])$. We note two interesting observations: 1) the influenza virus family is much

488 more connected (more red links) than the coronavirus family, possibly indicating that the 
489 influenza strains are less mutated than the coronavirus strains, and 2) SARS-CoV-1 has no direct 490 links to the influenza family.

492 This result supports our claims that SFA has the potential and efficiency to delineate the complex 493 mathematical structure of genetic sequences, and that it could become a useful tool in such 494 analyses. We need to stress here that, given the mathematics behind SFA, while we can make 495 direct comparisons of the complexity measure "C" within a certain family (where more or less 496 the number of bases is the same), we cannot compare complexities based on " $C$ " between 497 different families. This is due to the differences in nucleotide length between viral families. The 498 coronavirus family sequence length is approximately 30,450 bases, whereas the influenza family 499 sequence length is approximately 13,500 bases. As such SFA may "see" longer oscillations in 500 the coronavirus family than in the influenza family. Thus, there will be more entries above the 501 diagonal (in tables such as Table 2), and therefore, higher complexity in the coronavirus family.

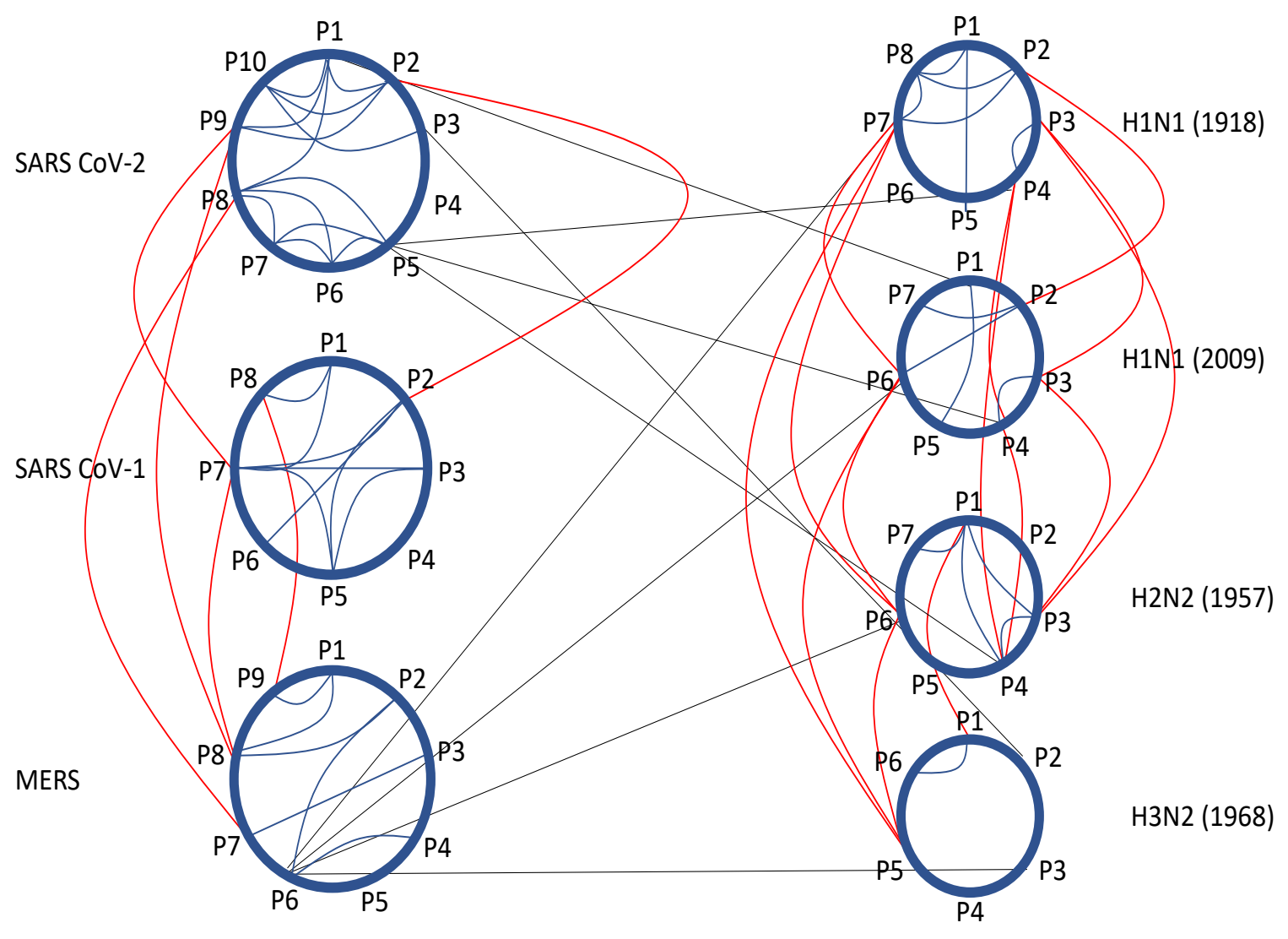


Figure 4: A complex network visualization of the relationships (connections) between individual

507 nucleotide sequences (blue), between sequences with in each individual family (the coronavirus

508 family and the influenza family; red), and between the two families (black) resulted from the

509 SFA. This picture is akin to structures of complex networks where in each community the nodes

510 are connected in a certain way (meaning the community obeys its own dynamics), but where

511 there are also connections between the communities.

514 Finally, it is interesting to note that the complexity measure " $\mathrm{C}$ " in the case of the coronavirus 515 family, relates to mortality and severity of symptoms as well as to the rate of transmission. As 516 "C" increases, the transmission rate to humans increases, but mortality rate decreases. It is 517 reported that symptoms of the SARS-CoV-2 are milder than SARS-CoV-1 and MERS, however, 518 the viral transmission rate (from human-to-human) is greater than the other family members. The 519 mortality rate of SARS-CoV-2 is lower (3.4\%) than that of SARS-CoV-1 (9.6\%) and MERS $520(35 \%)$ [20]. This relationship is not as clear, however, in the case of the influenza virus family. 521 Unfortunately, in this case the outbreaks span over a century and the actual numbers are skewed

522 by several factors such as: deaths by secondary infection (due to the unavailability of

523 antibiotics), hygiene, lack of experience and lack of proper healthcare, especially in the early 524 outbreaks, and other problems. For example, H1N1-1918 $(\mathrm{C}=6)$ infected 30\% of the planet's 525 population and $\mathrm{H} 1 \mathrm{~N} 1-2009(\mathrm{C}=4)$ infected $10 \%$ of the population. This is consistent with

526 "increasing $\mathrm{C} \rightarrow$ higher infection rate", but it's not consistent with "increasing $\mathrm{C} \rightarrow$ less mortality 527 rate". H1N1-1918 killed about 8\% of the infected, whereas H1N1-2009 killed only 0.0025\% of 528 the infected [21-28]. But how can we compare the conditions in 1918 and 2009? To complicate 529 comparisons further, there is hardly any reliable data of infection rates for H2N2 and H3N3. In 530 any case, the complexity measure "C" may hold a promise and could become a useful tool in the 531 prediction of transmission and mortality rates in future new viral strains.

\section{References}


(1982); 47:1099-1108.

[2] Ohno S. Codon preference is but an illusion created by the construction principle of coding sequences, Proc. Natl. Acad. Sci. USA (1998); 85:4378-4382.

542 [3] Yomo T, Ohno S. Concordant evolution and noncoding regions made it possible by the universal rule of TA/CG deficiency-TG/Ct excess, Proc. Natl. Acad. Sci USA (1989); 86:

545

[4] Tsonis AA, Elsner JB, Tsonis PA. Periodicity in DNA sequences: Implications in gene 8452-8456.

[5] Tsonis AA, Kumar P, Elsner JB, Tsonis PA. Wavelet analysis of DNA sequences, Phys. Rev. E (1996); 53:1828-1834.

[6] Lask AM. Introduction to Bioinformatics, $3^{\text {rd }}$ edition, Oxford University press (2008); 474 pp.

[7] Pevsner J. Bioinformatics and Functional Genomics, $3^{\text {rd }}$ edition, Willey-Blackwell (2015); $1124 \mathrm{pp}$.

[8] Wiskott L, Sejnowski TJ. Slow Feature Analysis: Unsupervised learning of invariance,

[9] Wiskott L. Estimating driving forces of nonstationary time series with slow feature analysis, (2003), http://arxiv.org/abs/cond-mat/0312317/

[10] Berkes P, Wiskott L. Slow feature analysis yields a rich repertoire of complex cells, Journal 566

567 [11] Blaschke T, Berkes P, Wiskott L. What is the relationship between slow feature analysis and 
independent component analysis? Neural Computation (2006); 18(10): 2495-2508, doi:10.1162/neco.2006.18.10.2495.

571 [12] Franzius M, Wilbert N, Wiskott L. Invariant object recognition and pose estimation with Slow Feature Analysis Neural Computation, (2011); 23(9):2289-2323, https://doi.org/10.1162/NECO_a_00171

[13] Yang P, Wang G, Zhang F, Zhou X. Causality of global warming seen from observations: a scale analysis of driving force of the surface air temperature time series in the Northern Hemisphere, Clim. Dyn. (2015); doi: 10.1007/s00382-015-2761-4.

[14] Tsonis AA, Pan X, Wang G, Nicolis C. On the min-max estimation of mean daily temperatures, Clim. Dyn. (2019); 53: 1981-1989, doi:10.1007/s00382-019-04757-6.

[15] Wiskott L et al. Slow feature analysis, Scholarpedia, (2011); 6(4): 5282.

[16] Torrence C, Compo GP. A practical guide to wavelet analysis, Bull. Amer. Meteor. Soc. (1998); 79: 61-78, doi:10.1175/1520-0477.

[17] Ng WM, Stelfox AJ, and Bowden TA. Unraveling virus relationships by structure-based phylogenetic classification, Virus Evolution (2020); 6(1), doi: 10.1093/ve/veaa003.

[18] Newman MEJ, Girwan M. Finding and evaluating community structure in networks, Phys. Rev. E (2004); 69: 026113, doi:10.1103/PhysRevE.69.26113.

[19] Newman MEJ. Modularity and community structure in networks, Proc. Natl. Acad. Sci. USA (2006);103: 8577-8582. pathogenesis with SRS-CoV and MERS-CoV infections, Future Virol. (2020); doi:10.2217/fvl-2020-0050. 
600 [21] Andreasen V, Viboud C, and Simonsen L. Epidemiologic Characterization of the 1918

601 InfluenzaPandemic Summer Wave in Copenhagen: Implications for Pandemic Control

602 Strategies, The Journal of Infectious Diseases (2008); 197 (2): 270-728.

603

604

[22] Cascella M, Rajnik M, Cuomo A, Dulebohn SC, and Di Napoli R. Features, Evaluation and Treatment Coronavirus (COVID-19).” In Statpearls [internet] (2020). StatPearls Publishing.

[23] Dawood FS, Iuliano AD, Reed C, Meltzer MI, Shay DK, Cheng P-Y, Don Bandaranayake D, et al. Estimated Global Mortality Associated with the First 12 Months of 2009 Pandemic Influenza A H1N1 Virus Circulation: A Modelling Study, The Lancet Infectious Diseases

[24] Hassan SA, Sheikh FN, Jamal S, Ezeh JK, and Akhtar. A. Coronavirus (COVID-19): A Review of Clinical Features, Diagnosis, and Treatment, Cureus (2020); 12 (3): e7355.

[25] Liu J, Xie W, Wang Y, Xiong Y, Chen S, Han, J and Wu. Q. 2020. A Comparative Overview of COVID-19, MERS and SARS: Review Article, International Journal of Surgery (2020); 81: 1-8.

[26] Taubenberger JK. The Origin and Virulence of the 1918 'Spanish' Influenza Virus, Proceedings of the American Philosophical Society (2006); 150 (1): 86-112.

[27] Viboud C, Grais RF, Lafont BAP, Miller MA, Simonsen L, and Multinational Influenza Seasonal Mortality Study Group. Multinational Impact of the 1968 Hong Kong Influenza Pandemic: Evidence for a Smoldering Pandemic, The Journal of Infectious Diseases (2005); 192 (2): 233-248. Impact of the 1957-1959 Influenza Pandemic, The Journal of Infectious Diseases (2016); 213(5): 738-745. 


\section{Declarations}

632

\section{Ethics approval and consent to participate}

634 Not applicable

635

636 Consent for publication

637 Not applicable

638

639 Data availability

640 All DNA sequences used in this analysis are public domain

642 Competing interests

643 The authors declare no competing interests

644

645 Funding

646 None

647

648 Author contribution

649 AAT designed the research, did some of the analysis, contributed to the interpretation of the

650 results, and wrote the first draft of the paper. GW, LZ and WL contributed largely to SFA

651 analysis. AK and KDLT contributed to interpretation of the results and writing of the manuscript.

652 All authors have approved the paper for submission. There are no competing interests.

653

654 Acknowledgements 
655 Not applicable

656

657 Author Information

658 Affiliations

659 Department of Mathematical Sciences, Atmospheric Sciences Group, University of 660 Wisconsin-Milwaukee, Milwaukee, WI 53201, USA, and Hydrologic Research Center, San 661 Diego, CA 92127, USA

662 Anastasios A. Tsonis

663 Key Laboratory of Middle Atmosphere and Global Environment Observation (LAGEO), 664 Institute of Atmospheric Physics, Chinese Academy of Sciences, Beijing 100029, China

665 Geli Wang Lvyi Zhang \& Wenxu Lu

666 Department of Biology and Center for Visual Sciences at Miami University, Oxford, OH 667 45056, USA

668 Aristotle Kayafas \& Katia Del Rio-Tsonis

669

670 Corresponding authors

671 Anastasios A. Tsonis aatsonis@uwm.edu

672 Katia Del Rio-Tsonis. delriok@miamioh.edu

673

674

675 
Figures

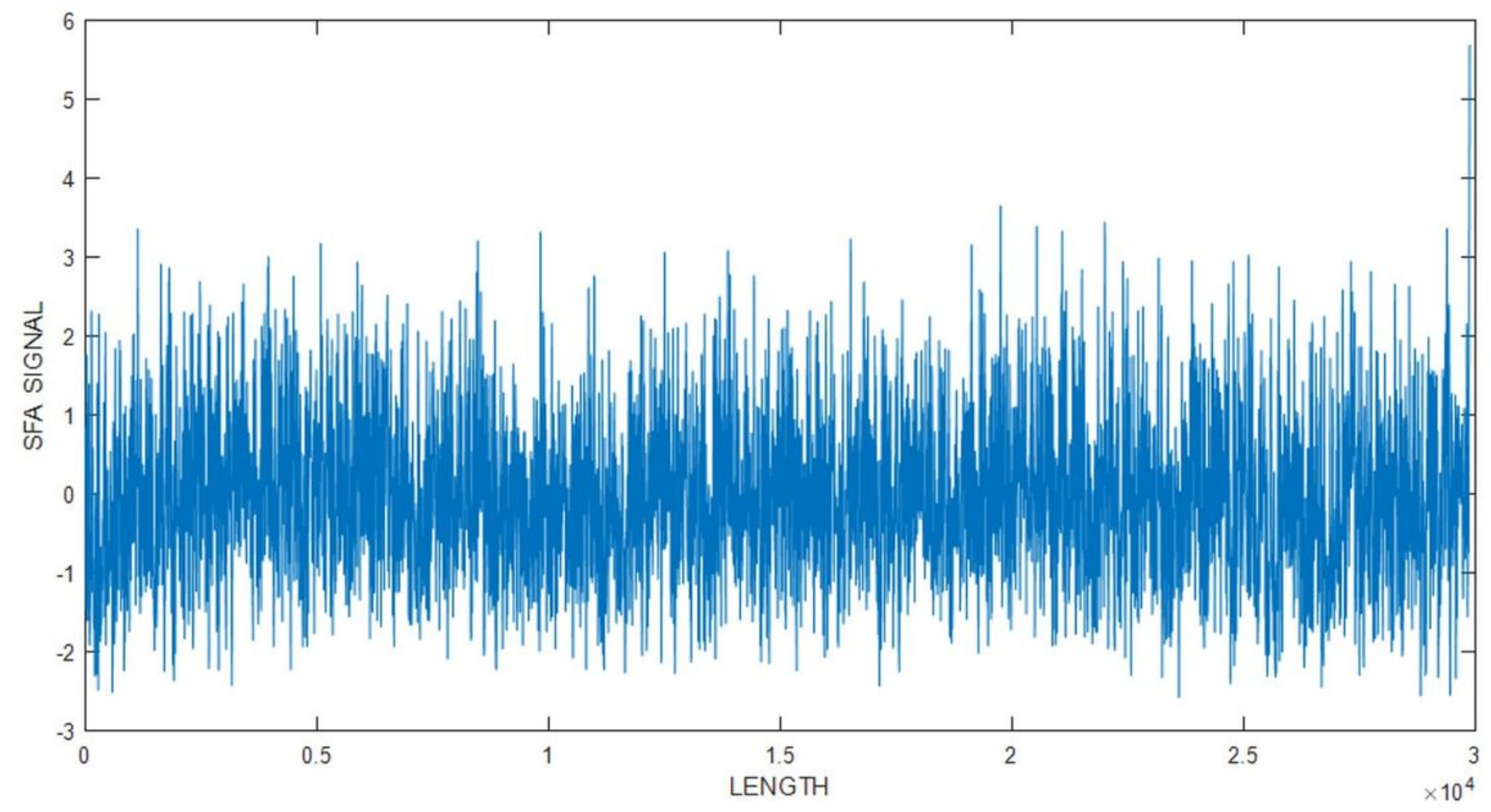

Figure 1

The SFA signal of the DNA sequence of SARS-CoV-2. Note the oscillatory components at many scales. 


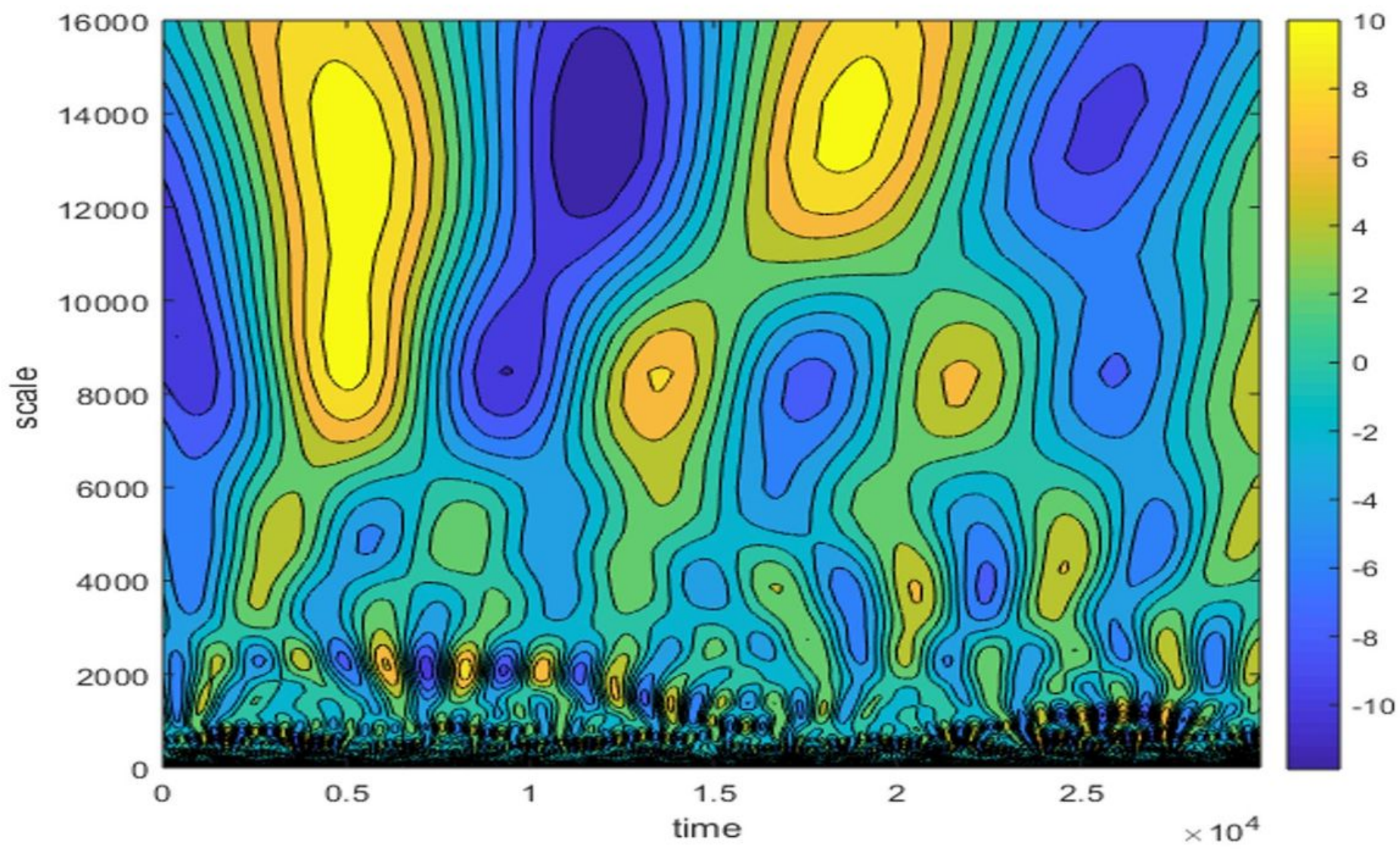

Figure 2

The wavelet of the signal extracted from Figure 1.

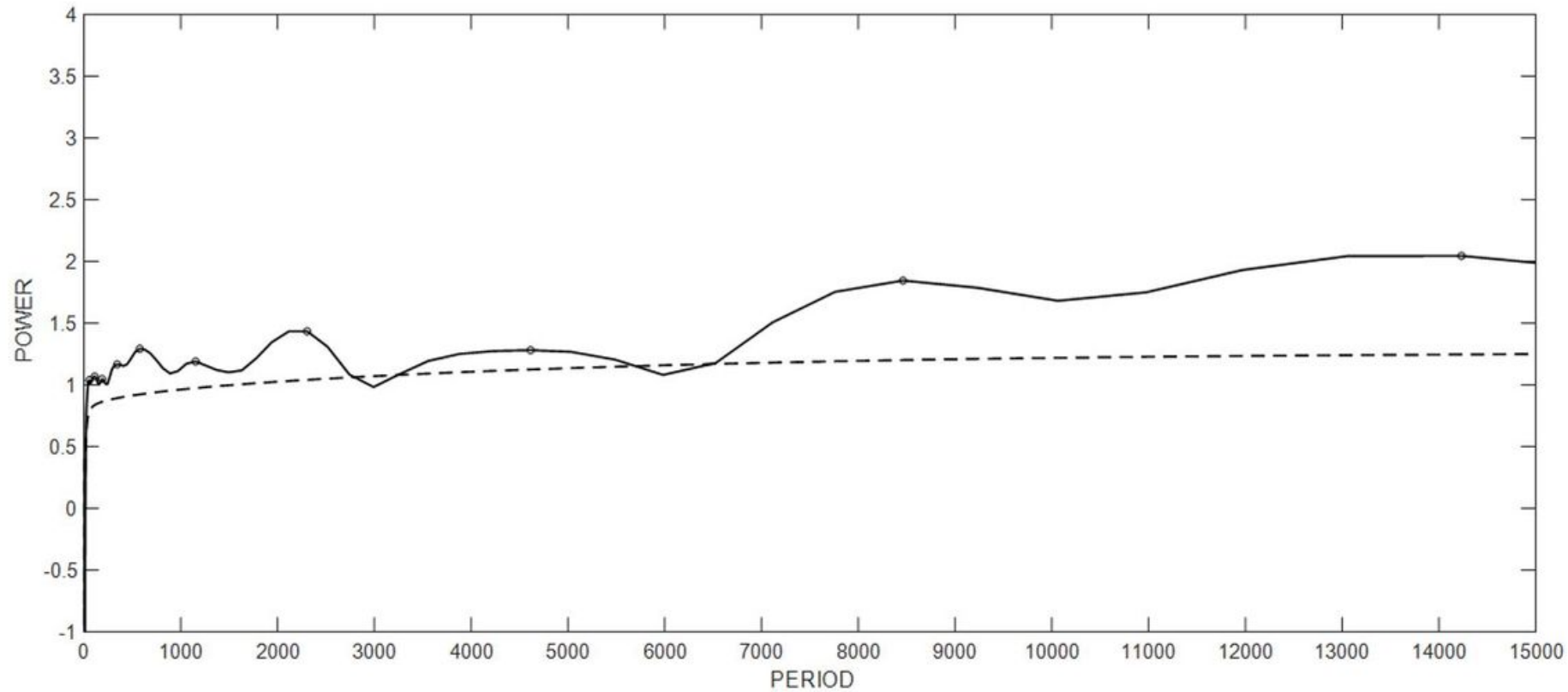

Figure 3 
The time-averaged power spectrum of the wavelet transform extracted from Figure 2. The dashed line represents the $95 \%$ confidence level. The dots show the periods of the oscillatory components of the driving force that are significant above the $95 \%$ level.

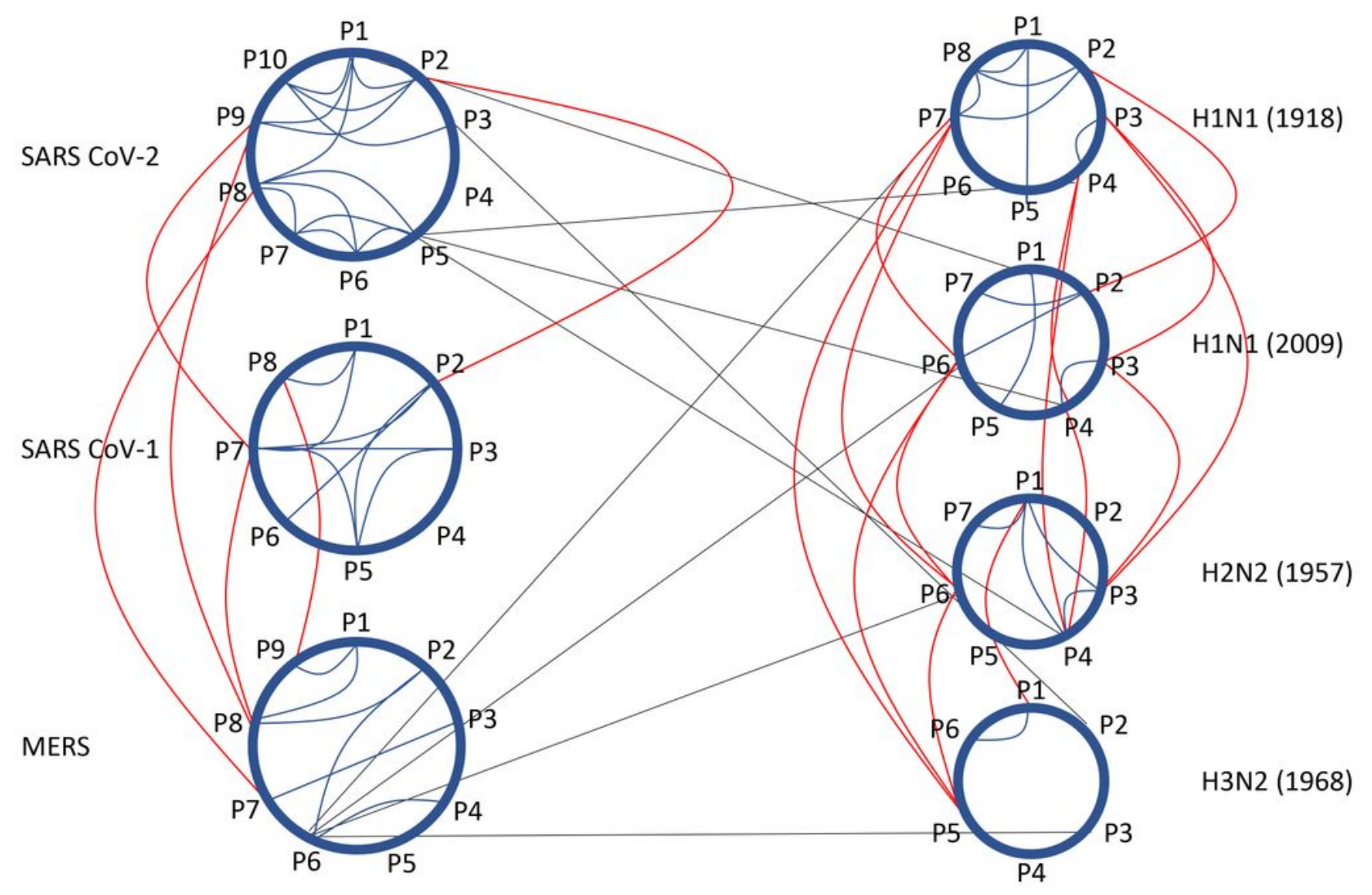

Figure 4

A complex network visualization of the relationships (connections) between individual nucleotide sequences (blue), between sequences with in each individual family (the coronavirus family and the influenza family; red), and between the two families (black) resulted from the SFA. This picture is akin to structures of complex networks where in each community the nodes are connected in a certain way (meaning the community obeys its own dynamics), but where there are also connections between the communities.

\section{Supplementary Files}

This is a list of supplementary files associated with this preprint. Click to download.

- Supplementarymaterial.docx 